\title{
ALUMNOS MEDIADORES. CONSTRUYENDO LA PAZ
}

STUDENT MEDIATION. MAKING PEACE

Rosaura Paulero *

Fecha de recepción: 6 mayo 2011.

Fecha de aceptación: 17 mayo 2011.

\begin{abstract}
Resumen
El presente artículo es producto de más de una década de trabajo en escuelas públicas, implementando programas de alumnos mediadores. Estos intervienen en con \ictos entre sus pares. A largo de todo el trabajo desarrollado se han formados más de 2.600 alumnos mediadores y capacitados más de 5.000 docentes. Las conclusiones que se presentan son el resultado de la recolección de testimonios e informes de los involucrados (alumnos, familias, docentes y directivos), y como consecuencia de esta experiencia es que podemos postular el carácter formativo y preventivo de la inclusión de la mediación en la escuela.
\end{abstract}

Palabras clave: alumnos mediadores, prevención, resolución.

\begin{abstract}
This article is the product of more than 10 years of work in public schools, using programs of students mediation. They take part in conflicts between them. Along all this work we have formed more than 2.600 students mediators and have receiving the knoweldge more than 5000 teachers. This conclusions are the result of the compilation of testimonies and reports of the envolved ones (students, familias, teachers and executives) and like a result of this experinece it is we can postulate the educative and preventive carachter to implemate the mediation in the school.
\end{abstract}

Key words: student mediation. prevention. resolution.

Licenciada en Psicología. Mediadora. Coordinadora equipos de mediadores escolares. Ministerio de Educación. Gobierno de la ciudad autónoma de Buenos Aires, Argentina.

Correo:rpaulero@ciudad.com.ar 


\section{El campo de trabajo}

Se dice que el conflicto es un rasgo inevitable de las relaciones sociales. El problema radica en que todo conflicto puede adoptar un curso constructivo o destructivo. Por lo tanto la cuestión no es eliminar el conflicto, sino asumirlo y enfrentarlo con recursos que nos permitan salir enriquecidos de esa situación.

El conflicto tiene muchas funciones y valores positivos. Evita el estancamiento, promueve el interés y la curiosidad, es la raíz del cambio personal y social, por consiguiente, ayuda a construir identidades, a conocernos mejor y a conocer a los demás.

La escuela, como espacio de encuentro de diferentes actores con multiplicidad de intereses y con diversidades culturales, sociales e individuales es generadora de diferencias y conflictos.

La intolerancia, la discriminación, las dificultades de comunicación se trasladan al aula y a la escuela. La crisis social y familiar hace que la escuela asuma funciones que históricamente no le estaban asignadas.

Partimos de considerar nuestro trabajo desde lo que se denomina metodológicamente investigación-acción. Lo fundamental se basa en la reflexión y comprensión sistemática de la práctica a efectos de mejorarla y transformarla. José Contreras Domingo sintetiza este proceso como "acción-observación-reflexión-nueva acción, etc.", por ello hay que entender el proceso de investigación-acción como un ciclo, un "bucle recursivo"1

\section{¿Por qué la mediación?}

La mediación es un proceso de comunicación por medio del cual un tercero imparcial, Ilamado mediador, ayuda a los participantes de un conflicto a dialogar sobre sus distintas perspectivas, explorar las emociones involucradas, reconocer sus intereses y necesidades y facilita la búsqueda de soluciones mutuamente satisfactorias.

Las características de la mediación son la voluntariedad, confidencialidad, imparcialidad, flexibilidad y autocomposición de las partes.

La mediación, como método de resolución de conflictos, ayuda a los miembros de la comunidad educativa a analizar y resolver sus conflictos desde perspectivas

Contreras, D. (1994) "Tema del mes: la investigación en la acción". España. Cuadernos de Pedagogía N2 224. 
cooperativas y positivas, atentas y respetuosas con los sentimientos e intereses de todos los involucrados.

Es por ello que en el año 1997 acepté la invitación para integrar un equipo interdisciplinario que se proponía realizar la primera experiencia local de Mediación Escolar, llevada adelante por un área del gobierno. Teníamos un esquema básico de trabajo. Nos planteábamos el carácter piloto de nuestra labor. Aún hoy, después más de 10 años de trabajo ininterrumpidos, seguimos conservando y reivindicamos el trabajo como artesanal. El equipo tiene como función capacitar y acompañar a la institución educativa que decida implementar un Programa de Alumnos Mediadores, trabajando como equipo externo a la institución educativa. La frecuencia de concurrencia a la escuela varía de acuerdo a la etapa del programa.

\section{Programa de alumnos mediadores}

Cuando comenzamos a trabajar implementando programas de alumnos mediadores en escuelas primarias y secundarias, nos basamos en experiencias de otras partes del mundo y en nuestra propia experiencia como mediadores.

Planteamos como objetivos de la inclusión de la mediación en el sistema educativo:

- Reconocer las capacidades para tratar los conflictos constructivamente.

- Adquirir habilidades comunicacionales y de negociación para facilitar la escucha respetuosa, el reconocimiento de la perspectiva del otro, el protagonismo y la creatividad en el abordaje constructivo de los conflictos.

- Promover la resolución de conflictos a través de métodos no adversariales y la disminución de los niveles de violencia, fortalecer los valores democráticos y de participación y mejorar la convivencia cotidiana.

\section{Definición y características}

Los programas de alumnos mediadores persiguen la creación de un equipo de mediadores integrado por alumnas y alumnos que son preparados para intervenir en conflictos entre sus pares.

Los alumnos son capacitados en abordaje colaborativo de conflictos y algunos de ellos son entrenados en mediación. Trabajan en comediación, de modo que las mediaciones son conducidas por un equipo.

Los programas de alumnos mediadores no sustituyen las sanciones establecidas frente a situaciones de indisciplina, sino que pueden complementarse con ellas. Si en 
el desarrollo de un conflicto interpersonal se producen faltas, deben ser sancionadas. Pero esta sanción no solucionará el problema, por lo tanto, es posible su derivación a mediación.

El fundamento de la mediación entre pares se basa en diferentes aspectos.

La mediación es un procedimiento jerárquico, los mediadores no ejercen poder sobre las partes ya que no deciden cómo termina el conflicto, sino que son los involucrados los que sí lo hacen. Por lo tanto, ambas partes deben hacerse responsables de la solución y por consecuencias del conflicto. Todo este proceso se facilita al ser el mediador un par, al manejar los mismos códigos comunicacionales y al estar seguras las partes que los mediadores no podrán ejercer poder sobre ellos. Esta creencia sería muy difícil de sostener si los mediadores fueran otros adultos de la institución escolar, dada su estructura piramidal. Asimismo, contribuye a desalentar la actitud paternalista de recurrir a un tercero adulto para que resuelva el conflicto.

El rol del docente, directivo o preceptor en la institución escolar hace necesario que el adulto se sienta impelido de dar una respuesta, una opinión, una solución, y así deben hacerlo en muchas situaciones. En cambio, los alumnos mediadores no sienten la misma presión, lo que les permite generar y sostener la tensión necesaria para que las partes se apropien no solo del conflicto, sino también de la construcción de la solución mutuamente satisfactoria que habrá de ponerle fin.

Finalmente, el principio de confidencialidad del proceso permite que el intercambio que se produce entre las partes sea sincero ya que nadie "deberá mentir" o "tergiversar las cosas" para ocultar una acción que podría ser vista como "reprochable o censurable" y, por consecuencia, sujeta a posterior sanción, si fuera reconocida en presencia de un adulto.

Desde 1997 se han desarrollado acciones dirigidas a difusión, capacitación, entrenamiento, seguimiento, supervisión y evaluación de la implementación de programas de mediación entre pares, asimismo, a poco de andar la realidad exigió llevar adelante intervenciones que implicaron la conducción de procesos de comunicación y de construcción de acuerdos, incluyendo tanto mediaciones como otros procesos colaborativos: generación de consenso u otros diseños de resolución de problemas.

Luego de más de diez años de trabajo y a pesar de que la situación social se observa día a día más compleja, constatamos que muchos de estos objetivos se han cumplido en las instituciones que han implementado con compromiso y dedicación programas de alumnos mediadores. De esto intentará dar cuenta el presente artículo.

Plantearé el marco normativo de la educación para la paz y la resolución cooperativa de conflicto, el marco pedagógico que sostiene el trabajo de campo, el modo particular de implementación de programas de alumnos mediadores y los resultados obtenidos. 


\section{Marco normativo}

El contenido de Educación para la Paz aparece planteado en el espíritu y la letra de la Ley Nacional de Educación ( $n^{\circ}$ 26.206).

ARTÍCULO $8^{\circ}$ - La educación brindará las oportunidades necesarias para desarrollar y fortalecer la formación integral de las personas a lo largo de toda la vida y promover en cada educando/a la capacidad de definir su proyecto de vida, basado en los valores de libertad, paz, solidaridad, igualdad, respeto a la diversidad, justicia, responsabilidad y bien común.

ARTÍCULO $11^{\circ}$ - c) Brindar una formación ciudadana comprometida con los valores éticos y democráticos de participación, libertad, solidaridad, resolución pacífica de conflictos, respeto a los derechos humanos, responsabilidad, honestidad, valoración y preservación del patrimonio natural y cultural.

ARTÍCULO $123^{\circ}$ - j) Desarrollar prácticas de mediación que contribuyan a la resolución pacífica de conflictos.

En un nivel más concreto se plasmaron los contenidos básicos comunes para el área de la Formación Ética y Ciudadana, acordados en el Consejo Federal de Educación. Dicha área cuenta con 5 ejes de formación:

\section{- Persona \\ - Valores \\ - Normas sociales \\ - Procedimientos \\ - $\quad$ Actitudes generales}

En cualquiera de estos ejes se encuentran contenidos relacionados con la Educación para la Paz.

Avanzando en el nivel de concreción encontramos que en los diseños curriculares correspondientes a los diferentes niveles se da lugar al trabajo con los valores de paz, solidaridad, justicia. Desde una apreciación general, como las planteadas en el marco general del nivel inicial, hasta la implementación institucional de programas de métodos pacíficos de resolución de conflictos como la mediación escolar, la escuela "se hace cargo" de este contenido necesario para la adquisición de habilidades sociales que favorezcan la convivencia armoniosa y pacífica.

A partir del segundo ciclo de la escuela primaria, los contenidos trabajados en Educación para la Paz se plantean como contenidos transversales, ya que brindan al alumno/a habilidades, conocimientos y actitudes que le serán de utilidad en su vida 
cotidiana escolar. Coincido con Zulema P. de Meaños ${ }^{2}$ en que “... la formación integral encuentra en esta propuesta de transversalidad de contenidos una solución que, por otra parte, puede aportar mucho a nuestra práctica educativa en cuanto a organización y globalización de contenidos y estrategias interdisciplinarias...".

\section{Inserción en la planificación escolar}

La transversalidad de los contenidos ofrece una solución metodológica que puede en algunos momentos parecer una dificultad. Si dichos contenidos no están en ninguna "materia", entonces no están en ningún lado. O, por el contrario, si están en todos lados, ya que aparecen en cada acción, no encuentran un momento específico para su tratamiento. Para evitar esta dispersión, Siede ${ }^{3}$ menciona cinco formatos diferentes y convergentes de incorporación a los procesos didácticos:

- Abordaje formativo de situaciones cotidianas: tomando hechos de la convivencia escolar y formulándolos como problemas, transformándolos en herramientas pedagógicas. En esta transformación es donde interviene el docente.

- Momento de enseñanza dentro de otras áreas: algunos contenidos de otras áreas, como, por ejemplo, las guerras en el área de Sociales, permiten la inserción de contenidos de FEyC.

- $\quad$ Proyectos específicos: son temas que requieren o justifican por la realidad social de la comunidad educativa un tratamiento especial. En esos casos se trabajan otorgándoles una carga horaria específica, en general involucran a más de un grupo-clase dentro de la escuela.

- Área de Conocimiento del Mundo, Primer Ciclo EGB: FEyC comparte el área con Sociales, Naturales y Tecnología.

- $\quad$ Aérea con carga horaria propia en el Tercer Ciclo EGB.

La Educación para la Paz encuentra cabida en cualquiera de las modalidades mencionadas. Considero que la implementación de un Programa de Mediación Escolar se encuentra en la tercera propuesta como proyecto específico.

De Meaños, Z. Los Contenidos Transversales, El Ateneo, 3ª ed. Bs. As.; 1999.

3 Siede, I. "Formación ética y ciudadana". Orientación general y proyecto formativo del área. Documentos. GCABA. 


\section{Elaboración de un proyecto}

Entendemos los proyectos transversales de Educación para la Paz como "un conjunto de actividades de enseñanza que buscan atender necesidades formativas de los estudiantes en el ámbito de prácticas actuales de la ciudadanía que demandan una respuesta de la escuela" ${ }^{4}$.

Entre sus propósitos se encuentran:

- Enseñar saberes, prácticas reflexivas y hábitos de vida que constituyan las bases para que los niños y niñas puedan integrarse a la sociedad, desde sus propios intereses y proyectos, para contribuir a mejorarla.

- Establecer modos institucionales de convivencia democrática que favorezcan la enseñanza de formas justas y solidarias de ejercicio del poder.

- Fomentar actitudes y hábitos de cuidado y defensa de la dignidad personal, de solidaridad y compromiso con los otros, de justicia en la resolución de conflictos y cooperación en proyectos comunes, de autonomía en la toma de decisiones y en la elección de modos de vida.

- El desafío: ¿Cómo pasar de la enseñanza de valores por sus meros enunciados a una enseñanza en valores?

- Los mediadores sabemos que la mediación nos plantea el desafío de trabajar con valores como la solidaridad, responsabilidad, compromiso, participación y el respeto. La mediación insta al ejercicio cotidiano de la cooperación y reconoce la interdependencia que existe entre los seres humanos.

- $\quad$ Recordemos que J. Piaget ${ }^{5}$ plantea que dado que la cooperación es un método, es difícil ver cómo podría cobrar existencia si no fuera mediante su propio ejercicio.

\section{Marco pedagógico}

Paulo Freire ${ }^{6}$ dice que la educación o bien hace un esfuerzo por domesticar a los estudiantes en conformidad a los valores y normas del grupo que manda o, bien para motivar a sus estudiantes para que sean creativos, responsables y libres. Siguiendo esta línea es que podemos plantear dos modelos de educación. Juan Pablo Lederach

4 Documento de Formación Etica y Ciudadana, Secretaría de Educación, Gobierno de la Ciudad de Buenos Aires.

5 Piaget, J. (1932), El Criterio Moral en el niño.

6 Citado por Lederach, J. y Chupp, M. (1995), ¿Conflicto y Violencia? Busquemos Alternativas Creativas Akron, PA. 
y Marcos Chupp, en Conflicto y Violencia...7, afirman que el modelo de educación transformadora debería orientarnos en nuestro trabajo en resolución de conflictos.

Para Freire, en la educación tradicional el sistema educativo se basa en un modelo parecido al bancario. Se percibe a los maestros como a los poderosos que poseen la información esencial. Los estudiantes, en cambio, son recipientes vacíos. El maestro consigna la información en los estudiantes para sacarla después del examen. Así que lo más importante es poner todo en la memoria, aunque el alumno no sepa cómo poder usarlo en su vida.

La educación transformadora se plantea que se puede aprender lo que Ilena una necesidad personal, también se puede aprender haciendo y en un contexto determinado que genere confianza.

El objetivo es crear una experiencia educativa, basada en problemas reales, con gente, un grupo que genere confianza, probando diferentes alternativas.

Los participantes de un sistema transformativo, son vistos como personas creativas, inteligentes y con la capacidad de actuar. La educación transformadora hace uso de muchas dinámicas, ejercicios, casos, juegos y experiencias a través de los cuales es posible crear un ambiente distinto, que permita una vivencia. Si uno se involucra en un proceso transformativo sobre la violencia y el conflicto, se tendrá que abandonar la concepción bancaria y tradicional de la educación que exige un papel pasivo a los alumnos.

\section{Etapas de un programa de mediación entre pares}

\section{Contacto inicial}

La implementación del trabajo en la escuela da comienzo con una etapa introductoria en la que se explora el interés de la comunidad educativa en el programa así como el compromiso de las autoridades.

El primer contacto institucional, a cargo de las coordinadoras, tiene como objetivo explicar las características del programa y los compromisos institucionales que se requieren para abordar la intervención. Asimismo, se explora el interés del equipo de conducción en el programa y su compromiso.

\section{Sensibilización}

El siguiente momento tienen como objetivo explicar el programa a docentes, administrativos y preceptores y evaluar, el apoyo e interés de los mismos.

7 Lederach, J y Chupp, M. (1995), ¿Conflicto y Violencia? Busquemos Alternativas Creativas Akron, PA. 
Esta actividad se desarrolla en una jornada de sensibilización organizada al efecto.

El requisito que tiene el equipo para desarrollar el programa es obtener un apoyo mayor al $80 \%$ de los participantes (los asistentes deben completar una encuesta anónima).

\section{Consolidación}

Una vez que el equipo decide implementar el programa en la institución, da comienzo a la etapa de gestación del proyecto.

El objetivo de esta etapa es construir con la comunidad educativa un programa a la medida de sus necesidades, respetando ciertas pautas básicas.

Las acciones que se desarrollan varían según las posibilidades de cada escuela, incluye una aproximación a la dinámica institucional mediante entrevistas y/o espacios compartidos por todos los miembros de la comunidad con el fin de integrar visiones, desafíos y propuestas así como generar compromiso con el programa.

\section{Capacitación}

El siguiente momento está centrado en la capacitación de los distintos integrantes de la escuela.

\section{A docentes, preceptores y directivos}

Se propone una capacitación de adultos de la comunidad en un curso introductorio en Resolución de Conflictos - Mediación que aborda la temática del conflicto, la comunicación, la negociación colaborativa y la mediación.

Los objetivos de dicha capacitación son:

* Favorecer la participación y el compromiso de los miembros de la comunidad educativa en el programa.

* Identificar el conflicto como un componente más de nuestras vidas reconociendo su valor positivo. Analizar distintos tipos de conflictos. Reconocer las capacidades para tratar los conflictos constructivamente.

* Adquisición de habilidades comunicacionales y en negociación colaborativa para: facilitar la escucha mutuamente respetuosa, el reconocimiento de la perspectiva del otro, el protagonismo y la creatividad en un abordaje constructivo de los conflictos.

* Formar a los docentes como capacitadores de alumnos, transformándolos en multiplicadores de la experiencia, favoreciendo así la apropiación del programa y el desarrollo del mismo en forma autogestora. 
Una vez finalizada esta etapa de capacitación de docentes, preceptores, directivos, etc., se vuelve sobre el diseño del programa con la finalidad de ajustarlo a la próxima etapa de acuerdo con el nuevo contexto generado tras la capacitación de adultos.

\section{Taller de padres}

Durante este etapa se abordan también talleres de padres en los que se les informa sobre el programa y se los introduce en temas como conflicto y comunicación. La participación en estos talleres depende de las características de la institución, nivel de enseñanza y difiere según las peculiaridades de cada "grupo padres".

\section{A alumnos}

En la capacitación introductoria de alumnos participa todo el curso, está a cargo de uno a más docentes de ese curso. Tiene una duración que varía según las características de la escuela y de cada grupo áulico e incluye temas similares a la capacitación brindada a los docentes. La metodología utilizada se basa en dinámicas que incluyen actividades lúdicas, ejercicios prácticos, juegos de rol, etc.

Los objetivos son: promover la reflexión y revisión de actitudes, la adquisición de nuevas habilidades con el propósito de mejorar la comunicación y prevenir situaciones de violencia, mejorando la convivencia cotidiana no solo en la escuela, sino en todos los ámbitos presentes y futuros.

Durante la capacitación de alumnos se brinda un espacio de tutoría a los docentes en la que el equipo externo funciona como apoyo con el objetivo de evacuar dudas y ayudar en el diseño y/o modificación de las actividades previstas.

\section{Selección de mediadores}

Al finalizar la capacitación introductoria, se lleva a cabo la selección de alumnos mediadores, la metodología de selección varía también de acuerdo a cada experiencia, sin embargo, todos incluyen la autopropuesta de los interesados, la exploración de la opinión de los compañeros y el aval de docentes y directivos.

\section{Entrenamiento}

Finalmente la etapa de capacitación finaliza con el entrenamiento de alumnos mediadores. La duración de los entrenamientos varían de acuerdo a las posibilidades de cada institución y el nivel de la misma, en general el equipo recomienda utilizar alrededor de 10 horas reloj para el nivel primario y 16 horas reloj para el nivel secundario.

Este entrenamiento lo imparte el equipo externo junto con algún docente de la escuela, que asesora a la institución. 
Se incluye entre sus contenidos: las características de la mediación, el rol del mediador, las reglas del proceso y se los prepara para el manejo de este: creación de contexto, exploración de perspectivas, emociones, intereses y necesidades, generación de opciones, selección de soluciones y construcción de acuerdos. El entrenamiento se basa en dinámicas participativas y juegos de rol.

\section{Puesta en marcha del centro de mediación}

Esta etapa comienza con la graduación de los alumnos mediadores en un acto del que participa toda la comunidad educativa con el objetivo de dar a conocer la puesta en marcha del centro de mediación y de legitimar el rol de los mediadores.

El centro se organiza con parejas de comediadores ya que los alumnos mediadores siempre trabajan de a dos, horarios, turnos, espacios, temas que se incluyen o excluyen, modalidades de referencia de mediaciones, etc.

Durante esta etapa también se encaran acciones dirigidas a la publicidad y difusión del servicio de mediación entre pares.

\section{Seguimiento y supervisión}

El seguimiento y supervisión de los alumnos mediadores se realiza en un primer momento en reuniones semanales que complementan la capacitación.

Al finalizar la mediación, los mediadores alumnos completan un formulario que se utiliza con fines estadísticos.

Se realizan reuniones en donde se trabajan las dificultades del rol y las de la implementación. Estas reuniones no se hacen exclusivamente con alumnos mediadores, sino también con docentes y directivos. Las mediaciones escolares asimismo se rigen por el principio de confidencialidad, por ende, el proceso no se realiza ante la presencia de adultos, ni con posterior información sobre el contenido del caso a estos. Sin embargo, esto no implica que no exista un monitoreo sobre la práctica de los mediadores, ya que cuentan con un espacio de supervisión a cargo de un adulto especialmente preparado para tal función.

Finalmente esta etapa puede incluir distintos momentos (por lo general una vez al año) donde se profundiza la capacitación de los mediadores

\section{Evaluación de la experiencia}

Durante el proceso de implementación se desarrollan distintos tipos de evaluación de las etapas (cuestionarios, encuestas, informes estadísticos de las mediaciones, etc.). Sobre el final de la intervención del equipo externo se realiza una evaluación del programa que incluye instrumentos como entrevistas con los distintos actores de la comunidad educativa, cuestionarios autoadministrados, evaluaciones estadísticas. 


\section{Resultados cuantitativos}

Cantidad de capacitados a diciembre de 2008.

- $\quad$ Alumnos mediadores: 2.611

_ Alumnos capacitados en resolución de conflictos: 20.292

- $\quad$ Docentes, preceptores y directivos: 5.185

- $\quad$ Talleres con padres: 540

Los informes de mediaciones presentados por los alumnos mediadores.

\section{Conclusiones}

- Se destacan como principales motivos de mediación los insultos (20,6\%), las burlas $(16,6 \%)$ y los malentendidos $(15,4 \%)$.

- $\quad$ El 65\% de los participantes en las mediaciones eran mujeres.

- En el $46 \%$ de los casos, los participantes de las mediaciones no mantuvieron una charla previa a la misma.

- Como motivos para solicitar la mediación se destacan la necesidad de amigarse de las partes (32\%) y el consejo de un docente $(26 \%)$.

- $\quad$ El 85\% de las mediaciones se resolvió con acuerdo.

- Según los mediadores, la mediación resultó muy satisfactoria o satisfactoria para los participantes en el $82,1 \%$ de los casos.

\section{Resultados cualitativos}

Las conclusiones que se presentan a continuación son resultado de la recolección de testimonios e informes de los involucrados (alumnos, familias, docentes y directivos).

Como resultado de esta experiencia es que podemos postular el carácter formativo y preventivo de la inclusión de la mediación en la escuela.

Teniendo en cuenta las características de imparcialidad, voluntariedad y protagonismo de las partes no podemos pensar que el mediador "enseña", no lo hace de manera directa pero sí en forma secundaria.

La implementación de programas de resolución de conflictos y mediación entre pares posee carácter formativo ya que los resultados observados en las distintas experiencias tienen características de aprendizaje:

- No son imputables a procesos madurativos.

- Son duraderos y modifican las estructuras de los participantes.

- Preparan dichas estructuras para nuevas adquisiciones. 
- El relevamiento de la experiencia nos aporta múltiples situaciones o anécdotas donde se reflejan estos enunciados teóricos.

- Podemos identificar cuatro sujetos de cambio o aprendizaje.

1. Los alumnos mediadores

2. Los alumnos que son parte de mediaciones

3. Los alumnos que reciben capacitación en resolución de conflictos

4. La institución escolar-incluyendo a los adultos-

1. Los cambios más notorios se observan en los alumnos mediadores. Adquieren habilidades para el reconocimiento y manejo de conflictos, la comunicación y la conducción de procesos, a la vez que ayuda a desarrollar su empatía y a poner en acción sus aprendizajes. Todo ello les permite mejorar su capacidad de expresarse verbalmente, ser más tolerantes y flexibles. Aceptan más fácilmente el punto de vista del otro.

La formación y el rol favorece su autoestima: se sienten útiles. Por lo tanto, promueve el compromiso y la participación. Esto implica que en la institución participan en muchas otras actividades.

Permite que reconozcan mejor sus emociones.

Algunos chicos que eran muy tímidos pudieron integrarse mejor al grupo.

Algunos de los Ilamados líderes negativos pudieron encontrar un rol positivo.

Por ejemplo, una docente nos comentó que al salir de la escuela, se encontró en una plaza cercana a dos alumnos que eran eternos rivales que estaban compartiendo amablemente un juego. Uno de ellos era mediador. Luego, al conversar con él, manifestó que se daba cuenta que era más tolerante y que ahora podía compartir espacios con algunos chicos que no eran sus amigos y con los cuales quizás no tenía mucho en común.

En los monoblocks de Villa Lugano, un barrio carenciado del sur de la ciudad, los chicos se agrupan de acuerdo a las filas de las torres de edificios, A, B, C, etc. En una escuela media vespertina había un centro de alumnos mediadores, ellos nos comentaron como cosa muy natural que los enfrentamientos entre filas o bandas de filas eran muy frecuentes y violentos. Como en varias filas había chicos mediadores, ellos actuaban como negociadores para evitar esos enfrentamientos.

De parte de las familias también hemos recibido comentarios al respecto. Algunos padres observan que sus hijos a partir de ser mediadores pueden ser más tolerantes con hermanos menores o establecer espacios de comunicación diferentes. Una mamá nos dijo que su hijo (alumno mediador) le dijo: "Mamá, quiero hablar con vos, 
si ahora no me podés escuchar, está bien, pero avísame cuándo vas a poder, porque necesito hablar con vos".

2. Pero también se observan cambios en los alumnos que participan de mediaciones. Participar del proceso les permite: experimentar una manera respetuosa de hablar y ser escuchado, de expresar necesidades y sentimientos. Incrementando la capacidad para reconocer al otro. Pueden encontrar un espacio donde el pensamiento crítico y la toma de decisiones son necesarios y valorados. Aceptar la responsabilidad de sus acciones y recurrir a la creatividad para pensar nuevas maneras de convivencia.

En una escuela primaria un alumno asistía como parte a mediación en forma recurrente. Los alumnos mediadores lo habían apodado el VIP (very important people). En una mediación se trabajó el tema de la amistad y los mediadores realizaron preguntas reflexivas. A partir de allí, su modo de relacionarse cambió y mejoró la convivencia. No asistió más a mediaciones por ese año.

3. Alumnos que reciben capacitación en resolución de conflictos. Por otro lado, relevamos constantemente la opinión de los docentes que refieren que a partir de la introducción de los contenidos de resolución de conflictos, se genera en el aula un mejoramiento del clima, una mejor convivencia. Esto a su vez produce una disposición favorable para la tarea del aprendizaje. Se prioriza el diálogo, por lo tanto disminuyen las situaciones de violencia o agresión.

Un ejemplo es una escuela primaria donde las docentes de $5^{\circ}$ grado habían trabajado con sus alumnos en resolución de conflictos. Históricamente existían discusiones entre los dos grupos de $5^{\circ}$ porque tenían que compartir un patio. Ese año, cuando terminaron el curso de resolución de conflictos, los alumnos se acercaron a sus maestras y les pidieron reunirse todos para acordar un plan para usar el patio. La reunión se realizó con la facilitación de las docentes y el acuerdo al que Ilegaron lo cumplieron.

En otra escuela los alumnos mediadores hicieron una reunión entre sus compañeros para elegir si usaban buzo o campera de egresado.

4. A nivel institucional, en relación con los adultos, los cambios son más difíciles, si bien no con la misma frecuencia que con los alumnos, también los docentes capacitados refieren cambios personales.

Muchos docentes cuentan que cuando se presentan situaciones en las que se sienten maltratados verbalmente por sus alumnos, pueden actuar distinto. Se toman unos minutos, le proponen al alumno que también se los tome en cuenta, luego conversan y generan un espacio de mayor colaboración.

Directores que plantean que los problemas ya no llegaban a su despacho. 
Docentes que se sorprenden del diálogo que se puede entablar con los alumnos y del poder de reflexión de los chicos.

Se ha observado que la implementación de programas de alumnos mediadores en sus diferentes etapas, generan un mejoramiento del clima institucional. Por ejemplo: en esta institución los docentes se habían capacitado en su totalidad en un curso intensivo durante el receso escolar, y todos de una y otra manera estaban trasladando su capacitación al aula. En aquella reunión los docentes comentaron que el clima escolar se observaba más tranquilo, y el equipo de conducción expresó que tenían la impresión que habían realizado menos llamados al SAME (Servicio de Urgencias Médicas).

\section{Comparativo de la intervención de1 SAME 1999-2001 (1er. cuatrimestre) en cantidad de intervenciones}

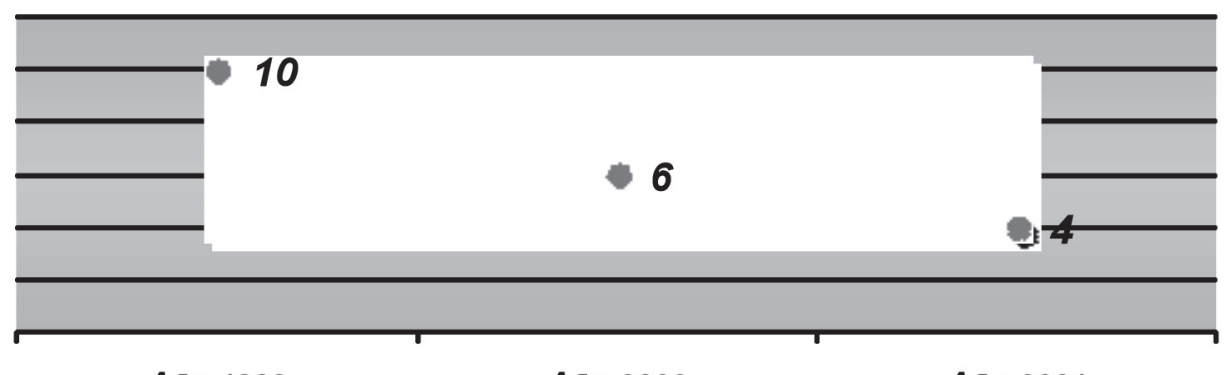

An̄o 1999

Año 2000

Año 2001

\section{Conclusiones}

Prevenir es llegar antes para que algo no suceda, es evitar, impedir, advertir e informarse. Por ello aprender es prevenir.

De la investigación realizada se concluye que para que estos resultados se den se deberán cumplir con las siguientes características:

- La implementación de un Programa de Alumnos Mediadores debería ser a pedido de la escuela y formar parte del proyecto institucional. Se deberá contar con un consenso o con una mayoría que acepte y promueva el trabajo en el programa. Asimismo, la participación de los alumnos en los espacios de mediación sea como mediadores o partes también tiene que tener como requisito la voluntariedad.

- Para poder implementar este tipo de programas, resulta esencial brindar capacitación a todos los actores institucionales (directivos, docentes, auxiliares, 
alumnos y padres). La formación de docentes como capacitadores de alumnos, con espacios de tutorías, los transforma en multiplicadores de la experiencia y permite el desarrollo desde la autogestión del programa.

- $\quad$ Fuerte compromiso con la tarea de docentes y directivos.

Pero, sobre todo, la mediación escolar constituye un importante trabajo preventivo y formativo, tanto a nivel individual, como colectivo, en tanto permite que los protagonistas de los conflictos tengan la oportunidad de:

- Vivirlo como una oportunidad para examinar diferentes perspectivas.

- Reconocer el impacto de sus respectivas acciones y responsabilizarse por ellas.

- Identificar emociones propias y ajenas.

- Explorar distintos intereses y necesidades.

- Trabajar cooperativamente en la búsqueda de soluciones que sean buena para todos los participantes.

\section{Bibliografía}

Datos estadísticos del Programa de Mediación Comunitaria. Área mediación escolar. GCABA. Argentina.

Girard, K.; Koch, S. (1998) Resolución de conflicto en las escuelas. España. Granica.

GottheIL, J.; Schiffrin, A. (compiladores) (1996) Mediación: una transformación en la cultura. Buenos Aires. Paidós.

Contreras, D. (1994) "Tema del mes: la investigación en la acción". España. Cuadernos de Pedagogía N2 224.

De Meaños, Z. (1999) Los Contenidos Transversales. El Ateneo, $3^{\text {a }}$ ed. Bs.As.

SiedE, I. "Formación ética y ciudadana". Orientación general y proyecto formativo del área. Documentos. GCABA

PiAGET, J. (1932) El Criterio Moral en el niño.

Lederach, J.; Chupp, M. (1995) ¿Conflicto y Violencia? Busquemos Alternativas Creativas, Akron, PA. 